\title{
Direct Observation of Ultrafast Exciton Localization in an Organic Semiconductor with Soft X-ray Transient Absorption Spectroscopy
}

\author{
D. Garratt ${ }^{*}$, L. Misiekis ${ }^{1}$, D. Wood ${ }^{1}$, E. W. Larsen ${ }^{1}$, M. Matthews ${ }^{1}$, O. Alexander ${ }^{1}$, P. Ye ${ }^{1}$, S.

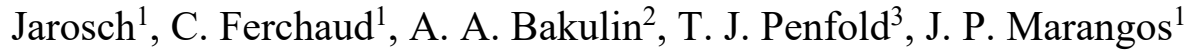 \\ ${ }^{1}$ Quantum Optics and Laser Science Group, Blackett Laboratory, Imperial College London, \\ London, SW7 2BW, UK. \\ ${ }^{2}$ Department of Chemistry and Centre for Processable Electronics, Imperial College London, \\ London, SW7 2BW, UK. \\ ${ }^{3}$ Chemistry - School of Natural and Environmental Sciences, Newcastle University, \\ Newcastle upon Tyne, NE1 7RU, UK \\ *Correspondence to: d.garratt15@imperial.ac.uk
}

\begin{abstract}
We report the first demonstration of time-resolved X-ray absorption spectroscopy to track previously undetected photoinduced dynamics of a paradigmatic crystalline conjugated polymer: poly(3-hexylthiophene) (P3HT) commonly used in solar cell devices. The $\pi \rightarrow \pi^{*}$ transition, the first step of solar energy conversion, is pumped with a $15 \mathrm{fs}$ optical pulse and the dynamics are probed by an attosecond soft X-ray pulse at the carbon K-edge. We observe direct spectroscopic signatures of the initially hot excitonic state, which is delocalized over multiple polymer chains, undergoing a rapid evolution on a sub $50 \mathrm{fs}$ timescale which can be directly associated with cooling and localization to form the lowest excitonic state on a single polymer chain. This sensitivity of time-resolved X-ray spectroscopy to the primary electron dynamics occurring directly after excitation paves the way for new insights in a wide range of organic optoelectronic materials.
\end{abstract}




\section{Introduction}

Energy capture through light induced processes in organic systems is at the heart of attempts to reduce our reliance upon unsustainable fuel sources using emerging clean energy technologies from artificial photosynthesis to organic solar cells. Photoexcitation of these molecular crystals and macromolecules generates tightly bound molecular excitons which are more localized and less prone to dissociation than excitonic states in inorganic semiconductors (1-4). Despite this, efficient charge photogeneration can be achieved in organic bulk heterojunction solar cell devices $(5,6)$. Optical and theoretical studies on these materials have suggested that this is due to electronic and vibronic coupling phenomena promoting a delocalization of the initially excited state in the donor, which enhances the efficiency of charge transfer and separation $(7,8)$. This effect is pronounced in the $\pi$ conjugated polymer poly(3-hexylthiophene) (P3HT), the subject of this work, where lamellae stacked crystalline regions in the thin film (9) enhances the delocalization between polymer chains $(10,11)$. After excitation, coupling of the electronic wavefunction to the vibrational and torsional modes of the polymer is assumed to lead to a rapid localization of the exciton (12-14) on ultrafast (sub $100 \mathrm{fs)} \mathrm{timescales.} \mathrm{This} \mathrm{is} \mathrm{detrimental} \mathrm{for} \mathrm{the} \mathrm{efficiency} \mathrm{of} \mathrm{solar} \mathrm{cell}$ devices using this material, therefore developing experimental methodologies which can study the effect are crucial for optimising the performance of these devices. However, probing these localization processes directly is not possible with existing ultrafast optical techniques $(15,16)$ both due to the lack of sensitivity to the spatial structure of the excited state wavefunction and the extremely short timescales often involved.

We address this problem by using element specific, soft X-ray transient absorption spectroscopy to directly probe ultrafast exciton localization in a conjugated polymer. X-ray absorption spectroscopy (XAS) is sensitive to the spatial extent of the valence wavefunction due to the localized nature of the core hole. Furthermore, the screening of the core-hole potential and therefore the core level energy is dependent on the oxidation state of the absorbing atom and leads to absorption features which strongly depend on the chemical environment of the atom. This sensitivity has been demonstrated with picosecond studies of photovoltaic materials using synchrotron radiation $(17,18)$, but these X-ray sources lack the temporal resolution required to capture highly transient states often important in solar energy conversion. This has recently changed with the advent of attosecond soft X-ray sources from high harmonic generation (19-24), and free electron lasers (25). Pioneering works using these sources have demonstrated time-resolved XAS spectroscopy (TR-XAS) of small gas phase molecules at the carbon K-edge (26-30) and at the $\mathrm{M}$ and L edges of heavier elements in simple semiconductors and metals (31-33). In this work we extend the technique to photoexcited organic semiconductor materials which have so far proved technically challenging due to their high photosensitivity (leading to a low damage threshold), low thermal conductivity and the requirement to probe in the water window spectral range. We overcome this by using a high flux water window high harmonic source in combination with careful control of the pump pulse intensity and monitoring of sample damage and heating.

\section{Experimental Methods}

Figure 1A shows a schematic of the experimental methodology. Briefly, free standing P3HT films with uniform thickness are produced by spin coating. The thickness of the samples is optimized to $\sim 100 \mathrm{~nm}$ to maximise the signal to noise ratio of the measurement. The visible absorbance spectrum of our P3HT samples is presented in Fig. 1B. The spectrum shows a broad multi-hump peak centred at $2.25 \mathrm{eV}$ corresponding to the $\pi \rightarrow \pi^{*}$ transition in P3HT. On the low energy side of the absorption band two peaks are observed consistent with previous measurements. These are related to inter-chain excitations and have a complex nature due to 
$\mathrm{H}$ - and J- aggregation as well as vibronic states as discussed in detail in references $(9,34)$. The samples are pumped with a $15 \mathrm{fs}$ optical pulse, with a pulse energy of $\sim 80 \mathrm{~nJ}$, centred at the peak of the absorption band. An attosecond soft X-ray supercontinuum extending from $\sim 150$ to $\sim 350 \mathrm{eV}$ is then used to probe the ensuing dynamics at the carbon K-edge. Further details on the experimental methods can be found in the supplementary material.

A

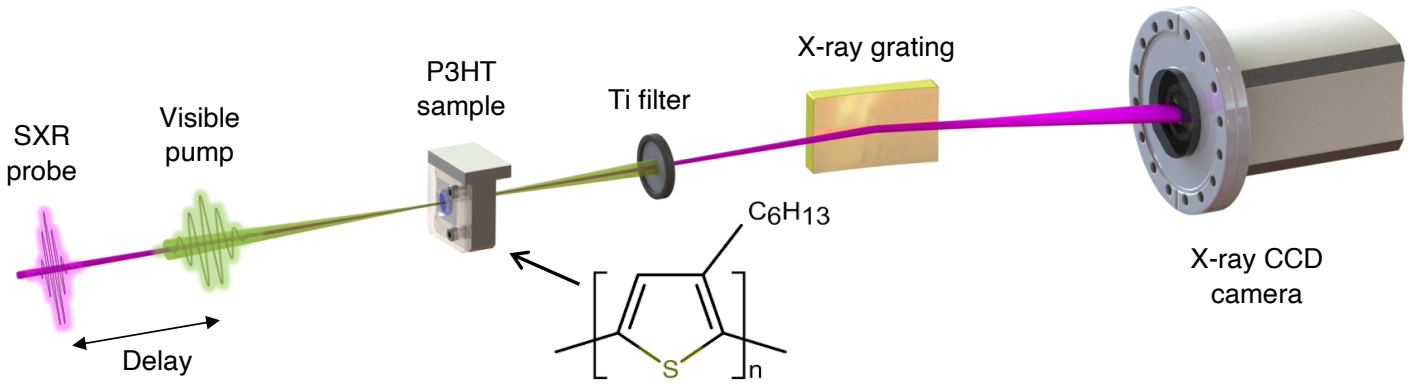

B

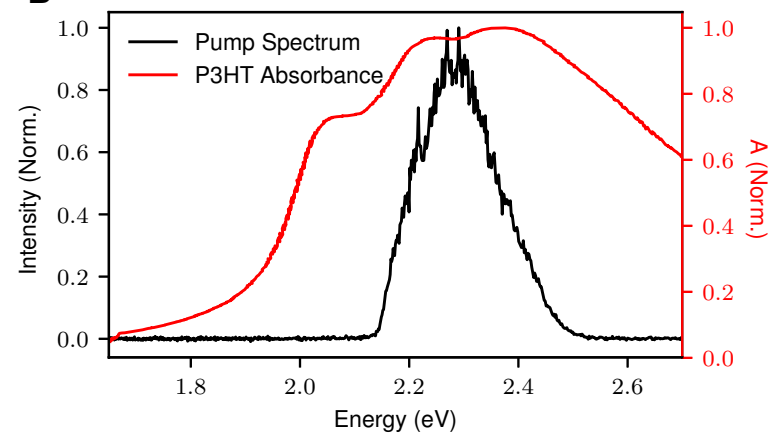

C

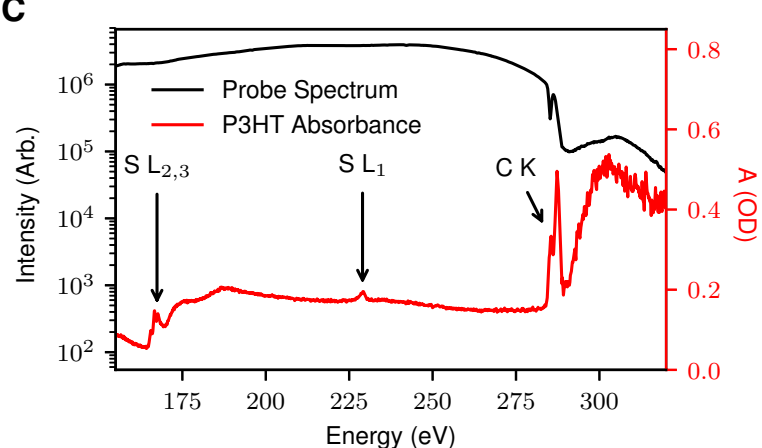

Fig. 1. (A) The experimental setup for time-resolved soft X-ray spectroscopy. A $15 \mathrm{fs}$ visible pump pulse excites the $\pi \rightarrow \pi^{*}$ transition in P3HT, and a temporally delayed attosecond soft Xray pulse probes the carbon and sulfur absorption edges. (B) The visible absorption spectrum of the P3HT samples used in this work, and the pump pulse spectrum which is centred at the maximum of the $\pi \rightarrow \pi^{*}$ resonance in P3HT. (C) A typical soft X-ray spectrum extending to $330 \mathrm{eV}$ (black line). The red line is the X-ray absorption spectrum of the P3HT sample, absorption features at the sulfur $\mathrm{L}_{1,2,3}$ and carbon K-edges are resolved simultaneously. The assignment of the absorption peaks are discussed in the main text.

A typical high harmonic spectrum is shown in Fig. 1C. Here the decrease in flux at around $284 \mathrm{eV}$ is due to absorption due to carbon contamination of the X-ray optics and filters. The absorption spectrum of the P3HT sample is plotted below. We simultaneously resolved the sulfur $\mathrm{L}_{2,3}, \mathrm{~L}_{1}$ and carbon K-edge at 165, 230 and $284 \mathrm{eV}$ respectively. The spectra are in good qualitative agreement with those published in the literature $(35,36)$. The three peaks at the sulfur $\mathrm{L}_{2,3}$ edge primarily correspond to spin-orbit split $\sigma^{*}(\mathrm{C}-\mathrm{S})$ orbitals with very weak contributions from the optically excited $\pi^{*}$ state. We have found that the absorption features at this edge are primarily sensitive to photoinduced sample damage and pump induced heating of the samples (see supplementary material), which do not manifest as delay dependent changes. We can exploit this and monitor the sample damage and heating at the sulfur L edge. Two pre-edge absorption peaks are observed at the carbon K-edge. The lowest energy peak at $285.5 \mathrm{eV}$ corresponds to the $\mathrm{C} 1 \mathrm{~s} \rightarrow \pi^{*}$ transition, where the highest sensitivity to the optically excited dynamics is expected. The higher energy peak at $287.5 \mathrm{eV}$ has 
contributions from $\sigma^{*}(\mathrm{C}-\mathrm{S})$ orbitals as well as $\sigma^{*}(\mathrm{C}-\mathrm{H})$ orbitals belonging to the alkyl side chains. This peak is therefore primarily sensitive to structural changes in the polymer. Above $290 \mathrm{eV}$ the X-ray absorption is saturated due to a combination of background carbon contamination and the strong absorption of $\mathrm{P} 3 \mathrm{HT}$ at these energies. This means that we do not resolve these peaks in the $\mathrm{P} 3 \mathrm{HT}$ absorption spectrum and instead focus on the lower energy features.

\section{Results}

We first present the results from pump probe studies at the carbon K-edge and then discuss how these relate to the ultrafast dynamics of the polymer. The differential absorption $(\Delta \mathrm{A})$ at the carbon K-edge following resonant photoexcitation of the $\pi \rightarrow \pi^{*}$ transition is shown in Fig. 2A Here, positive delays correspond to the X-ray pulse following the visible pump. From a singular value decomposition of the time dependent trace (37), we identify three significant components in the data. At short time delays ( $<30 \mathrm{fs}$, shown in Fig. 2B) three differential absorption features are visible. A decrease in absorbance around the maximum of the $\mathrm{C} 1 \mathrm{~s} \rightarrow \pi^{*}$ absorption peak $(285.5 \pm 0.4 \mathrm{eV})$ is accompanied by a weak increase in absorption, $1.2 \pm 0.5 \mathrm{eV}$ below the peak (referred to as feature A) and a stronger increase in absorption $0.5 \pm 0.4 \mathrm{eV}$ above the peak (feature B). Feature A corresponds to transitions into the vacant $\pi$ orbitals generated upon photoexcitation, while feature B corresponds to a narrowing and spectral blue shift of the $\mathrm{C} 1 \mathrm{~s} \rightarrow \pi^{*}$ peak giving rise to the derivative profile in the transient spectrum. The temporal dependence of the two positive differential absorption features is shown in Fig. 2B (bottom row). The temporal traces corresponding to features A and B are fitted with a Gaussian convolved with an exponential decay and a step function respectively. We find that feature $A$ is highly transient and decays with a time constant of $16 \pm 8$ fs while feature B persists for the $100 \mathrm{fs}$ of delay addressed in the measurement. The features are not fitted to a common time zero and we observe a small temporal offset of $9 \pm 4$ fs between the onset of feature B and feature A.
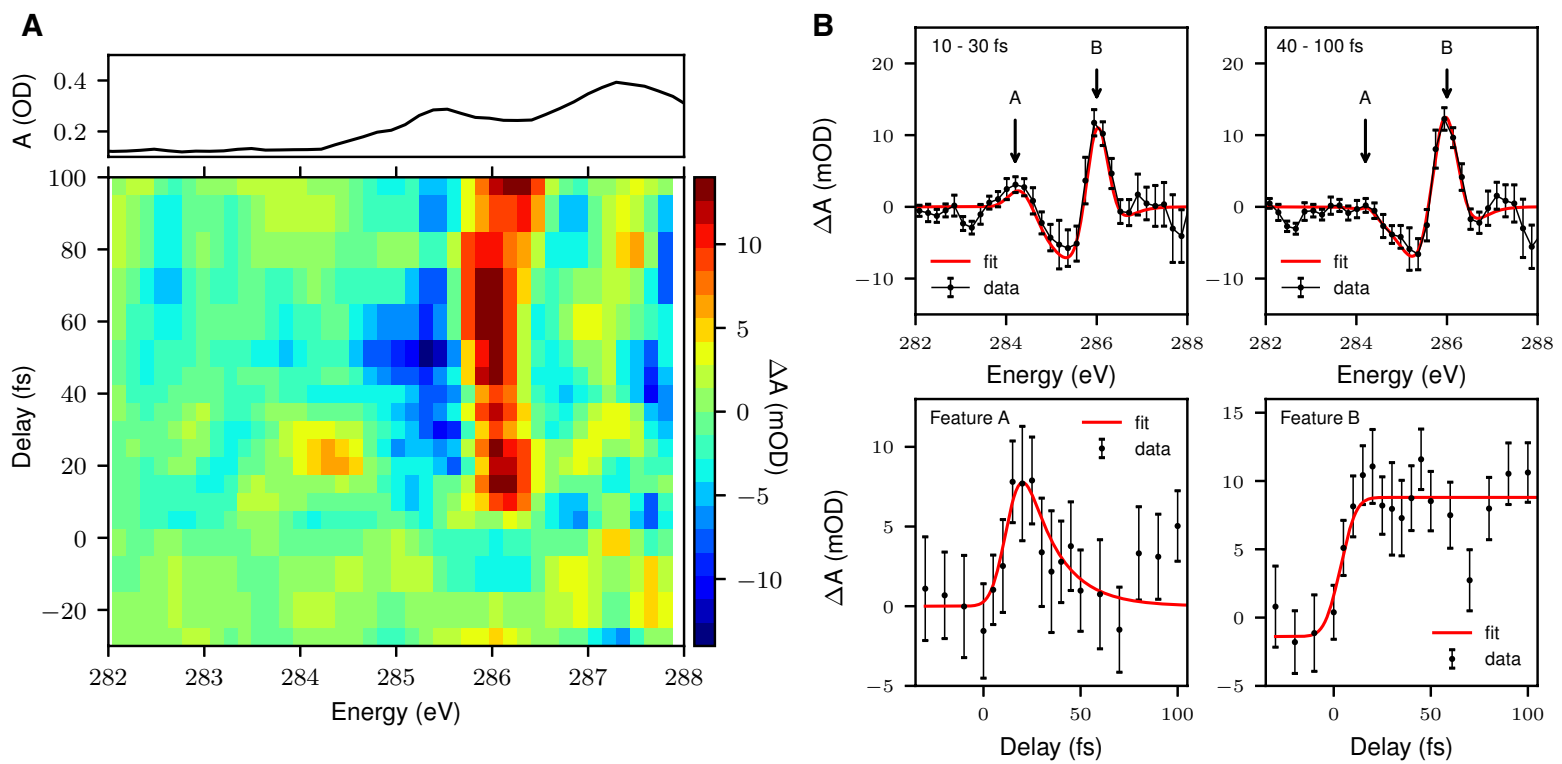

Fig. 2. (A) TR-XAS spectrum of P3HT from -30 fs to $100 \mathrm{fs}$ in the vicinity of the carbon Kedge. A static absorption spectrum of the sample is plotted above, on the same energetic axis. (B) The average TR-XAS spectrum across 10 fs to 30 fs and 40 fs to 100 fs (top row). The black lines show the measured spectrum and the red line is three component Gaussian fit to the data used to determine the spectral positions of the features discussed in the text. The bottom row shows the time evolution of the two positive differential absorption features $\mathrm{A}$ 
and $\mathrm{B}$, discussed in the main text. The red lines show an exponential decay and step function fit to the temporal traces for feature A and B respectively.

To interpret the experimental data, we perform simulations of the photoexcited XAS spectrum of a model oligothiophene system consisting of a single P3HT hexamer chain using Restricted Excitation Window Time-Dependent Density Functional Theory (REW-TDDFT) (38) within the approximation of the $\operatorname{PBE0}(39,40)$ exchange and correlation functional as implemented within the ORCA quantum chemistry package (41) (further details provided in the supplementary material). Fig. 3A (blue line) shows the simulated differential absorption spectrum for the lowest lying triplet excited state in this system, used to approximate the singlet exciton in P3HT, which has the same electronic structure. This represents a good approximation due to the lack of spin sensitivity of XAS spectroscopy as shown in ref (42). Here the theoretical signal is calculated from the difference of the ground and excited state XAS spectra. Despite the small size of the simulated system with respect to polymer (consisting of hundreds of repeat units) remarkably good agreement is found between the theoretical prediction and the main feature observed in the experiment at time delays $>40 \mathrm{fs}$. This derivative profile, as outlined above, corresponds to an edge shift suggesting a reduction in charge density on the carbon atoms after photoexcitation. This observation is confirmed using the electron density difference, $\rho_{\mathrm{T} 1}-\rho_{\mathrm{S} 0}$ for the model which shows a transfer of electron density away from the carbon and towards the sulfur atoms (Fig. 3B). We therefore assign the spectral narrowing and blue shift of the $\mathrm{C} 1 \mathrm{~s} \rightarrow \pi^{*}$ peak as a signature of the formation of the singlet exciton. The lack of significant time evolution of this peak across the $100 \mathrm{fs}$ of delay addressed in the experiment is consistent with the $\sim 200 \mathrm{ps}$ (43) lifetime of the exciton from optical studies.
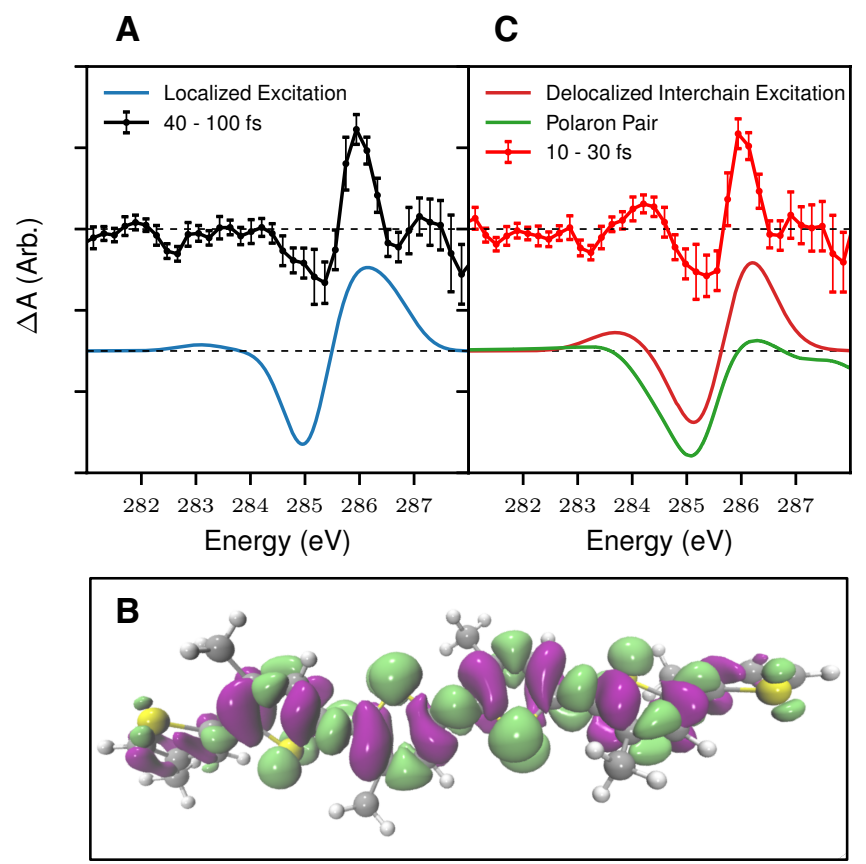

Fig. 3. (A) Comparison of the differential absorption signal at between 40 and 100 fs with the theoretical prediction for a $\mathrm{P} 3 \mathrm{HT}$ hexamer which approximates the singlet exciton localized on a single polymer chain. (B) The electron density difference between the simulated ground and excited states in the P3HT hexamer, purple indicates a loss of electron density and green indicates a gain. (C) A comparison between the differential absorption signal at short time 
delays (10 - $30 \mathrm{fs})$ and the theoretical differential absorption spectrum for a P3HT hexamer dimer, which approximates a delocalized interchain excitation the aggregated polymer and a cationic P3HT hexamer, which models a polaron pair in the polymer.

The theoretical transient spectrum shown in Fig. 3A contains a very weak positive differential absorption feature at around $283 \mathrm{eV}$, corresponding to a transition into the photoexcited hole in the $\pi$ orbital. However, although the spectral position of this increase approximately coincides with feature A identified in the experimental data at short time delays, it is significantly weaker. The calculated spectra also show a weak dependence on the nuclear geometry of the oligomer in this region of the spectrum (see supplementary material, Fig. S1) and therefore cannot be used to explain the experimental data.

There are three main processes which could be responsible for the discrepancy: Excitonexciton annihilation, polaron pair generation and localization processes. At the excitation densities used in this work $\left(0.29 \mathrm{~nm}^{-3}\right)$, the exciton-exciton annihilation rate is significant ( $\left.0.7 \times 10^{13} \mathrm{~s}^{-1}\right)$, but not enough to give rise to the $\sim 10 \mathrm{fs}$ timescale of the feature. In addition, this process leads to the transition of one exciton to a higher lying excited state (44) and would therefore be expected to lead to increased X-ray absorption at lower energies to what is observed in the experiment.

Polaron pair generation is also thought to occur on sub-100 fs timescales, and to be enhanced at high excitation densities. To investigate whether this would be consistent with the spectral features observed, we have modelled this by simulating the XAS spectrum of a cationic and anionic P3HT hexamer, as described in the supporting information. As shown in Fig. 3C, although this contains a derivative profile, like the experimental transient spectrum, it does not accurately reproduce the observed features, especially in intensity.

Localization effects present in the aggregated polymer are not captured in the simulated system shown in Fig. 3A as it contains only a single oligothiophene chain and therefore the effect of interactions between lamellae stacked polymer chains on the electronic structure are not included. Although complete description of the X-ray absorption in the highly extended polymer is beyond current theoretical methods, we model aggregation effects with two P3HT hexamers placed $0.9 \mathrm{~nm}$ apart (Fig. 3C). Crucially, although the shape of the derivative profile remains largely unchanged, this model leads to a significant increase in the intensity of the A feature, consistent with the experimental transient spectrum recorded at early times. This indicates that the A feature offers a direct measure of the extent of delocalization of the electron hole pair and its decay. This is because a delocalization of the excited electronic state between polymer chains gives an enhancement in the transition amplitude for the X-ray probe below the edge. Our calculations indicate that this is due to a subtle change in the electronic structure of the dimer, leading to an increase electron density at the carbon $2 p$ orbitals compared to the monomer, leading to extra strength for the $1 \mathrm{~s}-2 \mathrm{p}$ dipole transition observed in the measurement. The temporal evolution of the feature, which decays within 20 fs, is likely driven cooling and localization of the initially formed of hot exciton (45). This is consistent with the timescale reported by recent quantum dynamics simulations (13) and the timescale of fluorescence/absorption depolarisation from optical spectroscopies $(14,15)$. Our results give direct evidence that this is due to a localization of the excited state.

In conclusion, we have demonstrated the application of time-resolved X-ray absorption spectroscopy at the carbon K-edge to a prototypical crystalline homopolymer donor material in organic photovoltaics. We have observed the TR-XAS signature of the singlet exciton in 
this system and shown that the signal is sensitive to delocalization of the exciton between polymer chains. By combining this with the exceptional timing resolution of our soft X-ray and visible pump source, we have directly observed the localization of the interchain exciton in P3HT within 20 fs. These localization processes are driven by vibronic coupling of the electronic states in the polymer to the vibrational and torsional modes of the system. Our simulations suggest that the TR-XAS signal is also sensitive to nuclear motion in the polymer (see supplementary Fig. S1) and therefore future studies with moderate improvements in the signal to noise ratio will be able to unravel the role of vibronic coupling in the localization dynamics on femtosecond timescales. The technique presented here can also be extended to study the effect of localization charge transfer dynamics in Donor:Acceptor bulk heterojunction solar cells such as PM6:Y6 (46) or P3HT:PCBM, as well as photosynthetic light harvesting complexes. This can be achieved by probing at different elemental absorption edges, for example the K-edges of nitrogen and oxygen, to resolve the localization of charge at different sites in the system. Our results therefore open up a range of new possibilities in studying the influence of localization on charge transfer in organic photoconversion systems. 


\section{References}

1. A. Davydov, Theory of Molecular Excitons (Springer, 2013).

2. C. J. Bardeen, The structure and dynamics of molecular excitons. Annual Review of Physical Chemistry 65, 127-148 (2014).

3. W. Barford, Excitons in conjugated polymers: A tale of two particles. The Journal of Physical Chemistry A 117, 2665-2671 (2013).

4. J. Brebels, J. V. Manca, L. Lutsen, D. Vanderzande, W. Maes, High dielectric constant conjugated materials for organic photovoltaics. Journal of Materials Chemistry A 5, 24037-24050 (2017).

5. Y. Cui, H. Yao, J. Zhang, T. Zhang, Y. Wang, L. Hong, K. Xian, B. Xu, S. Zhang, J. Peng, Z. Wei, Over $16 \%$ efficiency organic photovoltaic cells enabled by a chlorinated acceptor with increased open-circuit voltages. Nature Communications 10, 1-8 (2019).

6. S. Liu, J. Yuan, W. Deng, M. Luo, Y. Xie, Q. Liang, Y. Zou, Z. He, H. Wu, Y. Cao, High-efficiency organic solar cells with low non-radiative recombination loss and low energetic disorder. Nature Photonics 14, 300-305 (2020).

7. S. M. Falke, C. A. Rozzi, D. Brida, M. Maiuri, M. Amato, E. Sommer, A. De Sio, A. Rubio, G. Cerullo, E. Molinari, C. Lienau, Coherent ultrafast charge transfer in an organic photovoltaic blend. Science 344, 1001-1005 (2014).

8. A. De Sio, F. Troiani, M. Maiuri, J. Réhault, E. Sommer, J. Lim, S. F. Huelga, M. B. Plenio, C. A. Rozzi, G. Cerullo, E. Molinari, Tracking the coherent generation of polaron pairs in conjugated polymers. Nature Communications 7, 1-8 (2016).

9. H. Sirringhaus, P. Brown, R. Friend, M. M. Nielsen, K. Bechgaard, B. LangeveldVoss, A. Spier-ing, R. A. Janssen, E. Meijer, P. Herwig, D. M. De Leeuw, Twodimensional charge transport in self-organized, high-mobility conjugated polymers. Nature 401, 685-688 (1999).

10. F. Paquin, G. Latini, M. Sakowicz, P. L. Karsenti, L. Wang, D. Beljonne, N. Stingelin, C. Silva, Charge separation in semicrystalline polymeric semiconductors by photoexcitation: is the mechanism intrinsic or extrinsic? Physical Review Letters 106, 197401 (2011).

11. J. Clark, C. Silva, R. H. Friend, F. C. Spano, Role of intermolecular coupling in the photophysics of disordered organic semiconductors: aggregate emission in regioregular polythiophene. Physical Review Letters 98, 206406 (2007).

12. K. H. Park, W. Kim, J. Yang, D. Kim, Excited-state structural relaxation and exciton delocalization dynamics in linear and cyclic pi-conjugated oligothiophenes. Chemical Society Reviews 47, 4279-4294 (2018).

13. R. Binder, D. Lauvergnat, I. Burghardt, Conformational dynamics guides coherent exciton migration in conjugated polymer materials: First-principles quantum dynamical study. Physical Review Letters 120, 227401 (2018).

14. W. Popp, M. Polkehn, R. Binder, I. Burghardt, Coherent charge transfer exciton formation in regioregular $\mathrm{p} 3 \mathrm{ht}$ : A quantum dynamical study. The Journal of Physical Chemistry Letters 10, 3326-3332 (2019). 
15. N. Banerji, S. Cowan, E. Vauthey, A. J. Heeger, Ultrafast relaxation of the poly (3hexylthiophene) emission spectrum. The Journal of Physical Chemistry C 115, 9726 (2011).

16. M. M. L. Grage, Y. Zaushitsyn, A. Yartsev, M. Chachisvilis, V. Sundström, T. Pullerits, Ultrafast excitation transfer and trapping in a thin polymer film. Physical Review B 67, 205207 (2003).

17. F. G. Santomauro, J. Grilj, L. Mewes, G. Nedelcu, S. Yakunin, T. Rossi, G. Capano, A. Al Haddad, J. Budarz, D. Kinschel, D. S. Ferreira, Localized holes and delocalized electrons in photoexcited inorganic perovskites: Watching each atomic actor by picosecond x-ray absorption spectroscopy. Structural Dynamics 4, 044002 (2017).

18. F. Roth, M. Borgwardt, L. Wenthaus, J. Mahl, S. Palutke, G. Brenner, G. Mercurio, S. Molodtsov, W. Wurth, O. Gessner, W. Eberhardt, Direct observation of charge separation in an organic light harvesting system by femtosecond time-resolved XPS. Nature Communications 12, 1196 (2021)

19. A. S. Johnson, D. R. Austin, D. A. Wood, C. Brahms, A. Gregory, K. B. Holzner, S. Jarosch, E. W. Larsen, S. Parker, C. S. Strüber, P. Ye, J. W. G. Tisch, J. P. Marangos, High flux soft x-ray harmonic generation from ionization-shaped few-cycle laser pulses. Science Advances 4, eaar3761 (2018).

20. S. M. Teichmann, F. Silva, S. Cousin, M. Hemmer, J. Biegert, 0.5-kev soft x-ray attosecond continua. Nature Communications 7, 1-6 (2016).

21. T. Popmintchev, M. C. Chen, D. Popmintchev, P. Arpin, S. Brown, S. Ališauskas, G. Andriukaitis, T. Balčiunas, O. D. Mücke, A. Pugzlys, A. Baltuška, B. Shim, S. E. Schrauth, A. Gaeta, C. Hernández-García, L. Plaja, A. Becker, A. Jaron-Becker, M. M. Murnane, H. C. Kapteyn, Bright coherent ultrahigh harmonics in the kev X-ray regime from mid-infrared femtosecond lasers. Science 336, 1287-1291 (2012).

22. D. Popmintchev, B. R. Galloway, M. C. Chen, F. Dollar, C. A. Mancuso, A. Hankla, L. Miaja-Avila, G. O'Neil, J. M. Shaw, G. Fan, S. Ališauskas, G, Andriukaitis, T. Balčiunas, O. D. Mücke, A. Pugzlys, A. Baltuška, H. C. Kapteyn, T. Popmintchev, M. M. Murnane, Near-and extended-edge X-ray absorption fine-structure spectroscopy using ultrafast coherent high-order harmonic supercontinua. Physical Review Letters 120, 093002 (2018).

23. C. Schmidt, Y. Pertot, T. Balciunas, K. Zinchenko, M. Matthews, H. J. Wörner, J. P. Wolf, High-order harmonic source spanning up to the oxygen k-edge based on filamentation pulse compression. Optics Express 26, 11834-11842 (2018).

24. N. Ishii, K. Kaneshima, K. Kitano, T. Kanai, S. Watanabe, J. Itatani, Carrier-envelope phase-dependent high harmonic generation in the water window using few-cycle infrared pulses. Nature Communications 5, 1-6 (2014).

25. J. Duris, S. Li, T. Driver, E. G. Champenois, J. P. MacArthur, A. A. Lutman, Z. Zhang, P. Rosenberger, J. W. Aldrich, R. Coffee, G. Coslovich, F. Decker, J. M. Glownia, G. Hartmann, W. Helml, A. Kamalov, J. Knurr, J. Krzywinski, M.-F. Lin, J. P. Marangos, M. Nantel, A. Natan, J. T. O’Neal, N. Shivaram, P. Walter, A. Li Wang, J. J. Welch, T. J. A. Wolf, J. Z. Xu, M. F. Kling, P. H. Bucksbaum, A. Zholents, Z. Huang, J. P. Cryan, A. Marinelli, Tunable isolated attosecond X-ray pulses with gigawatt peak power from a free electron laser. Nature Photonics 14, 30-36 (2020). 
26. Y. Pertot, C. Schmidt, M. Matthews, A. Chauvet, M. Huppert, V. Svoboda, A. Von Conta, A. Tehlar, D. Baykusheva, J.-P. Wolf, H. J. Wörner, Time-resolved x-ray absorption spectroscopy with a water window high harmonic source. Science $\mathbf{3 5 5}$, 264-267 (2017).

27. K. Schnorr, A. Bhattacherjee, K. J. Oosterbaan, M. G. Delcey, Z. Yang, T. Xue, A. R. Attar, A. S. Chatterley, M. Head-Gordon, S. R. Leone, O. Gessner, Tracing the 267 $\mathrm{nm}$-induced radical formation in dimethyl disulfide using time-resolved $\mathrm{x}$-ray absorption spectroscopy. The Journal of Physical Chemistry Letters 10, 1382-1387 (2019).

28. A. Bhattacherjee, K. Schnorr, S. Oesterling, Z. Yang, T. Xue, R. de Vivie-Riedle, S. R. Leone, Photoinduced heterocyclic ring opening of furfural: Distinct open-chain product identification by ultrafast x-ray transient absorption spectroscopy. Journal of the American Chemical Society 140, 12538-12544 (2018).

29. A. Bhattacherjee, C. D. Pemmaraju, K. Schnorr, A. R. Attar, S. R. Leone, Ultrafast intersystem crossing in acetylacetone via femtosecond $\mathrm{x}$-ray transient absorption at the carbon k-edge. Journal of the American Chemical Society 139, 16576-16583 (2017).

30. A. R. Attar, A. Bhattacherjee, C. Pemmaraju, K. Schnorr, K. D. Closser, D. Prendergast, S. R. Leone, Femtosecond x-ray spectroscopy of an electrocyclic ringopening reaction, Science 356, 54-59 (2017).

31. M. Schultze, K. Ramasesha, C. Pemmaraju, S. Sato, D. Whitmore, A. Gandman, J. S. Prell, L. Borja, D. Prendergast, K. Yabana, D. M. Neumark, Attosecond band-gap dynamics in silicon. Science 346, 1348-1352 (2014).

32. M. Volkov, S. A. Sato, F. Schlaepfer, L. Kasmi, N. Hartmann, M. Lucchini, L. Gallmann, A. Rubio, U. Keller, Attosecond screening dynamics mediated by electron localization in transition metals. Nature Physics 15, 1145-1149 (2019).

33. M. Zürch, H.-T. Chang, L. J. Borja, P. M. Kraus, S. K. Cushing, A. Gandman, C. J. Kaplan, M. H. Oh, J. S. Prell, D. Prendergast, C. D. Pemmaraju, Direct and simultaneous observation of ultrafast electron and hole dynamics in germanium. Nature Communications 8, 1-11 (2017).

34. F. C. Spano, C. Silva, $\mathrm{H}$-and j-aggregate behavior in polymeric semiconductors. Annual Review of Physical Chemistry 65, 477-500 (2014).

35. B. Watts, S. Swaraj, D. Nordlund, J. Luning, H. Ade, Calibrated nexafs spectra of common conjugated polymers. The Journal of Chemical Physics 134, 024702 (2011).

36. A. Johnson, L. Miseikis, D. Wood, D. Austin, C. Brahms, S. Jarosch, C. Strüber, P. Ye, J. P. Marangos, Measurement of sulfur L-2, 3 and carbon K-edge XANES in a polythiophene film using a high harmonic supercontinuum. Structural Dynamics $\mathbf{3}$, 062603 (2016).

37. C. Ruckebusch, M. Sliwa, P. d. Pernot, A. De Juan, R. Tauler, Comprehensive data analysis of femtosecond transient absorption spectra: A review. Journal of Photochemistry and Photobiology C: Photochemistry Reviews 13, 1-27 (2012).

38. S. D. George, T. Petrenko, F. Neese, Time-dependent density functional calculations of ligand K-edge X-ray absorption spectra. Inorganica Chimica Acta 361, 965-972 (2008). 
39. J. P. Perdew, K. Burke, M. Ernzerhof, Generalized gradient approximation made simple. Physical Review Letters 77, 3865 (1996).

40. C. Adamo, G. E. Scuseria, V. Barone, Accurate excitation energies from timedependent density functional theory: Assessing the pbe0 model. The Journal of Chemical Physics 111, 2889-2899 (1999).

41. F. Neese, The orca program system. Wiley Interdisciplinary Reviews: Computational Molecular Science 2, 73-78 (2012).

42. S. Eckert, J. Norell, R. M. Jay, M. Fondell, R. Mitzner, M. Odelius, A. Föhlisch, T1 Population as the Driver of Excited-State Proton-Transfer in 2Thiopyridone. Chemistry A European Journal 25, 7 (2019).

43. J. Piris, T. E. Dykstra, A. A. Bakulin, P. H. V. Loosdrecht, W. Knulst, M. T. Trinh, J. M. Schins, L. D. Siebbeles, Photogeneration and ultrafast dynamics of excitons and charges in P3HT/PCBM blends. The Journal of Physical Chemistry C 113, 1450014506 (2009).

44. R. Tempelaar, T. L. Jansen, J. Knoester, Exciton exciton annihilation is coherently suppressed in h-aggregates, but not in j-aggregates. The Journal of Physical Chemistry Letters 8, 6113-6117 (2017).

45. T. R. Nelson, D. Ondarse-Alvarez, N. Oldani, B. Rodriguez-Hernandez, L. AlfonsoHernandez, J. F. Galindo, V. D. Kleiman, S. Fernandez-Alberti, A. E. Roitberg, S. Tretiak, Coherent exciton-vibrational dynamics and energy transfer in conjugated organics. Nature Communications 9, 1-9 (2018).

46. J. Yuan, Y. Zhang, L. Zhou, G. Zhang, H.-L. Yip, T.-K. Lau, X. Lu, C. Zhu, H. Peng, P. A. Johnson, M. Leclerc, Single-junction organic solar cell with over $15 \%$ efficiency using fused-ring acceptor with electron-deficient core. Joule 3, 1140-1151 (2019).

47. R. Trebino, Frequency-resolved Optical Gating: The Measurement of Ultrashort Laser Pulses (Springer Science \& Business Media, 2012).

48. G. Dicker, M. P. de Haas, L. D. Siebbeles, J. M. Warman, Electrodeless time-resolved microwave conductivity study of charge-carrier photogeneration in regioregular poly (3-hexylthiophene) thin films. Physical Review B 70, 045203 (2004).

49. J. C. Duda, P. E. Hopkins, Y. Shen, M. C. Gupta, Thermal transport in organic semiconducting polymers. Applied Physics Letters 102, 251912 (2013).

50. F. Weigend, Accurate coulomb fitting basis sets for h to rn. Physical Chemistry Chemical Physics 8, 1057-1065 (2006).

Acknowledgments We gratefully acknowledge invaluable scientific discussions with Jenny Nelson and James Durrant and thank Jenny Nelson for insightful comments on the manuscript. Assistance from Allan Johnson, Chris Brahms, Dane Austin, Alvaro SanchezGonzalez and Emma Simpson on the HHG source and the early development of the experimental apparatus are gratefully acknowledged as is the invaluable technical assistance from Andrew Gregory and Susan Parker. Funding: We acknowledge the UK Engineering and Physical Sciences Research Council for the funding of this work principally through grant number EP/R019509/1 and the MURI (EPSRC/DSTL) grant EP/N018680/1, and for 
earlier stages of the work EP/I032517/1 and the ERC Advanced Grant "ASTEX". DG and OGA acknowledge support from EP/L016524/1 


\section{Supplementary Material}

\section{Theoretical Comparisons and the Effect of Nuclear Motion}

The effect of nuclear geometry on the simulations is investigated further by comparison of the differential absorption signal in the tetrathiophene oligomer at fixed nuclear geometry and that where the excited state nuclear relaxation has been performed (see Fig. S1). The difference between the two transient signals is small and primarily manifests as a small spectral shift in the pre-edge differential absorption.

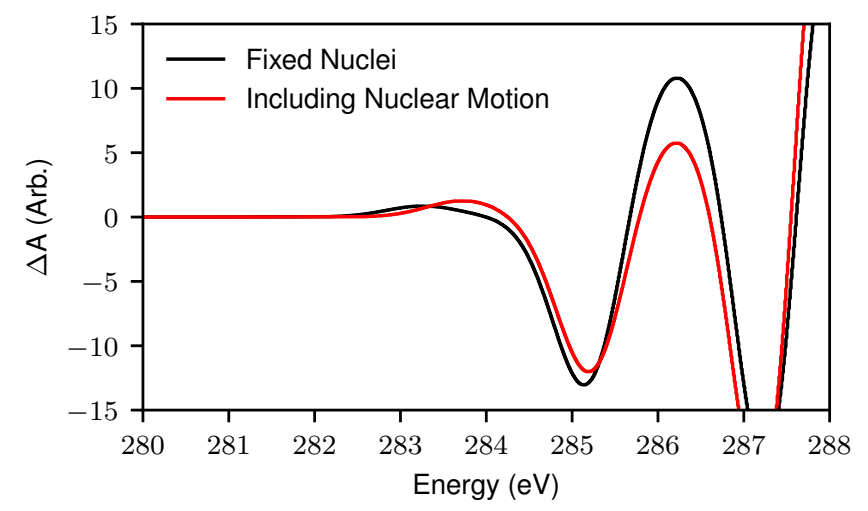

Fig. S1. The effect of including excited state nuclear motion on the differential absorption spectrum of the simulated thiophene hexamer.

\section{$\underline{\text { Experimental Methods }}$}

The soft X-ray source used in this work has been described in detail previously (12). Briefly, a commercial optical parametric amplifier is pumped with $8 \mathrm{~mJ}, 30 \mathrm{fs}$ pulses from a commercial Ti:Saphire laser system. The idler, which has a central wavelength of $1750 \mathrm{~nm}$, is compressed to $\sim 12 \mathrm{fs}$ using a hollow core fibre pulse compression system giving $\sim 600 \mu \mathrm{J}$ pulses with an excellent spatial profile. This is split into a pump and probe pulses using an annular mirror with a $1 \mathrm{~mm}$ hole. The majority of the pulse energy is reflected and used for soft X-ray high harmonic generation and $\sim 30 \mu \mathrm{J}$ is transmitted and used to generate the pump pulse. In the pump arm, the delay between the pump and probe is controlled with a piezoelectric stage with $1 \mathrm{~nm}$ accuracy. The visible pump pulse is then generated via cascaded second harmonic generation and sum frequency generation in a single $100 \mu \mathrm{m}$ thick beta barium borate (BBO) crystal. A half waveplate before the generation crystal ensures that the polarization of the pump pulse and soft X-ray probe are parallel. After the generation crystal, the third harmonic is spectrally filtered by 4 reflections from low dispersion dielectric mirrors before recombination with the soft X-ray probe at an annular mirror with a $3 \mathrm{~mm}$ hole. In the probe arm, the portion of the beam reflected by the annular mirror is focused with a $15 \mathrm{~cm}$ focal length CaF2 lens into a $1 \mathrm{~mm}$ length gas cell filled with 2.5 bar of $\mathrm{Ne}$, housed in a double differential pumping jacket, generating a soft X-ray supercontinuum extending to $\sim 330 \mathrm{eV}$. The driving laser field is then blocked by a $200 \mathrm{~nm}$ Ti filter and the soft X-ray beam passes through the centre of the annular recombination mirror. The pump and probe are focused onto the target with a $50 \mathrm{~cm}$ Au coated toroidal mirror to focal spot radii of $54 \pm 1$ $\mu \mathrm{m}$ and $40 \pm 2 \mu \mathrm{m}$ respectively. The X-ray spectrum is then spectrally dispersed by a concave, aberration corrected grating, with an effective line density of $1200 \mathrm{l} / \mathrm{mm}$, onto a thermoelectrically cooled CCD camera. The focal point of the harmonic radiation constitutes 
the source point for this spectrometer. A second $200 \mathrm{~nm}$ Ti filter, at the entrance to the spectrometer blocks the residual pump pulse not absorbed in the sample. The energy range of the spectrometer extends from $150 \mathrm{eV}$ to $350 \mathrm{eV}$ with an estimated spectral resolution $(\Delta \mathrm{E} / \mathrm{E})$ of $1.4 \times 10^{-3}$.

Temporal overlap between the pump and probe pulses is determined via spectral interferometry between the pump pulse and the third harmonic of the HHG driving laser generated in the gas cell. This enables time zero to be determined to an accuracy of $15 \mathrm{fs}$.

\section{Sample Preparation}

The free standing P3HT samples are produced by a bilayer deposition method. A clean glass substrate is first spin coated with a thin $(150 \mathrm{~nm})$ water soluble layer of polystyrenesulfonic acid (PSSA) which is dried for $10 \mathrm{~min}$ at $60^{\circ} \mathrm{C}$. Regioregular P3HT (molecular weight $=52$ $\mathrm{kDa}$ ), dissolved in dichlorobenzene with a concentration of $20 \mathrm{mg} / \mathrm{ml}$ is then spin coated on top of the water soluble layer at a spin speed of $1000 \mathrm{rpm}$. After drying for 10 minutes at 60 ${ }^{\circ} \mathrm{C}$, the samples are submerged in water to dissolve the PSSA layer and the P3HT layer is transferred to a stainless steel sample holder with $25,200 \mu \mathrm{m}$ drilled holes for transmission measurements. The thickness of the samples used in the measurements is estimated to be $\sim 100 \mathrm{~nm}$ from the X-ray absorption of the sample

\section{Data Analysis}

The static P3HT absorption spectra presented in Fig.1 are measured by integrating the soft X-ray spectrum for 5 minutes with and without the sample in the beam. The sample absorbance (A) is then calculated from the sample transmission spectrum $\mathrm{I}_{\text {sample }}(\mathrm{E})$ and the background spectrum, $\mathrm{I}_{\text {nosample }}(\mathrm{E})$ according to the Beer Lambert law, such that

$$
A_{\text {sample }}(E)=-\log _{10}\left(\frac{I_{\text {sample }}(E)}{I_{\text {no sample }}(E)}\right)
$$

Transient absorption spectra are obtained by recording P3HT transmission spectra with and without the pump pulse for a series of pump probe time delays. Each transmission spectrum is integrated for 1 minute. The pumped and unpumped X-ray transmission spectra are normalised to the harmonic flux at $250 \mathrm{eV}$ and then the differential absorbance is calculated according to

$$
\Delta A(E)=-\log _{10}\left(\frac{I_{\text {pumped }}(E)}{I_{\text {unpumped }}(E)}\right)
$$

where $\mathrm{I}_{\text {pumped }}$ and $\mathrm{I}_{\text {unpumped }}$ denote the sample transmission with and without the pump pulse. The signal is averaged over 15 repeated delay scans, and the error bars shown in Fig. 2 are estimated from the standard deviation of the repeated measurements. A two-dimensional Wiener filter is applied to the transient absorption spectrum to obtain the plots shown in Fig. 2. The temporal traces are obtained by integrating $0.5 \mathrm{eV}$ regions centered at each differential absorption peak. The integrated yield of the negative differential feature, is subtracted from each yield to give the temporal traces.

Pump Pulse $\underline{\text { Characterisation }}$ 
The pump pulse duration is characterised via second harmonic frequency resolved optical gating (SH-FROG) (47). Figure S2 shows the measured and reconstructed FROG spectrograms along with the reconstructed temporal and spectral intensity and phase of the pump. The measurement gives a pump pulse duration (FWHM) of $16 \mathrm{fs}$. There is some residual third order spectral phase which gives the small pre/post pulse observed in the temporal intensity profile. This is attributed to the third order phase accumulated by the fundamental due to transmissive optics before the generation crystal.

The pump focal spot size is measured to be $54 \pm 1 \mu \mathrm{m}$ (1/ $\mathrm{e}^{2}$ radius) from the transmission of the pump beam through a pinhole placed in the sample plane. This leads to a pump fluence of $870 \pm 60 \mu \mathrm{J} \mathrm{cm}^{-2}$ and an average excitation density across the probed sample volume of 0.29 $\mathrm{nm}^{-3}$. At the excitation densities employed here, exciton-exciton annihilation is thought to be a significant deactivation mechanism for the photoexcited exciton. Assuming an excitonexciton annihilation rate coefficient of $2.3 \times 10^{-8} \mathrm{~cm}^{3} \mathrm{~s}^{-1}(48)$, we estimate that this process reduces the singlet exciton lifetime from $200 \mathrm{ps}$ (43) to $0.6+4.4-0.35 \mathrm{ps}$, but this remains well outside our measured delay range and show is not captured in these measurements.
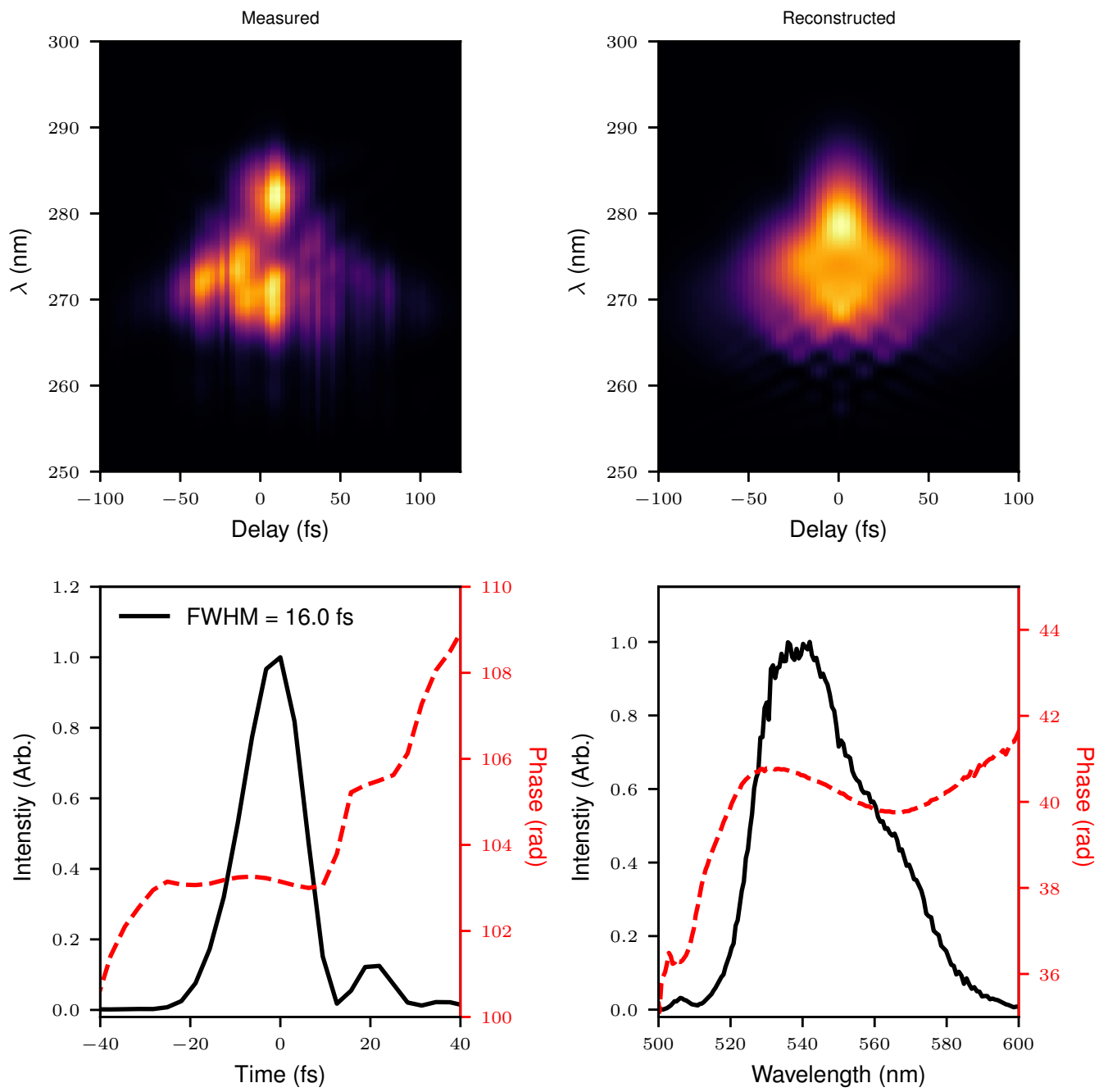

Fig. S2. The measured and reconstructed FROG spectrogram of the visible pump pulse along with the reconstructed temporal and spectral intensity and phase. The measurement gives a pulse duration of 16 fs (FWHM). 


\section{Sample Damage and Heating}

P3HT is a highly photosensitive material with low thermal conductivity $\left(\sim 0.2 \mathrm{Wm}^{-1} \mathrm{~K}(49)\right)$. Therefore, particular attention was paid to avoiding permanent damage to the sample by the pump pulse train and to characterising the sample temperature during the measurement. The damage threshold of the P3HT samples used in this work was determined to be $1.1 \pm 0.1 \mathrm{~mJ}$ $\mathrm{cm}^{-2}$. Above this pump fluence, photobleaching of the samples is observed. We also see a reduction in the magnitude of the absorption features at the sulfur $\mathrm{L}_{2,3}$ edge and carbon $\mathrm{K}$ edge indicating a structural change in the polymer. For the data presented in the main text (Fig. 2) we confirm that significant sample damage does not occur by comparing the sulfur $\mathrm{L}_{2,3} \mathrm{X}$-ray absorption before and after the measurement.

Due to the low thermal conductivity, heat conduction from the sample is not efficient so elevated sample temperatures are reached when irradiated by the pump pulse. To estimate the sample temperature during the pumped acquisitions, we simulate the temperature distribution of the sample for $\mathrm{CW}$ illumination of equivalent power and focal spot size (corresponding to $52 \mu \mathrm{W}$ of absorbed heat flux). The simulations include thermal conduction from the pumped volume and to the sample holder as well as black body cooling from the faces of the sample. Figure S3 shows the results of the temperature simulations. The average sample temperature across the probed volume of the sample is $180^{\circ} \mathrm{C}$. This is below the melting temperature for $\mathrm{P} 3 \mathrm{HT}$, and we do not see any permanent damage to the samples after performing the pump probe measurements. The elevated temperature during the pumped acquisitions is reversible and manifests as a delay independent background in the transient absorption spectrum. This is estimated by taking the average of the transient spectrum at negative time delays and subtracting this from all other delay points.

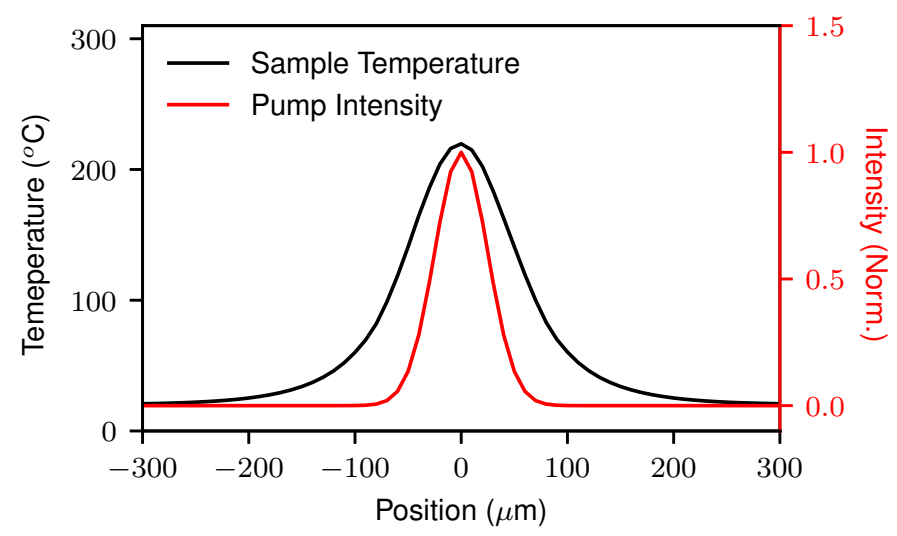

Fig. S3. The radial temperature distribution of the P3HT sample illuminated by an $80 \mu \mathrm{W}$ $\mathrm{CW}$ laser beam, corresponding to $52 \mu \mathrm{W}$ absorbed power (black line) along with the simulated pump intensity profile (red line). The average temperature across the radius of the probe pulse is $180^{\circ} \mathrm{C}$.

\section{Theoretical Methods}

All simulations were performed using the ORCA quantum chemistry package (41). The XAS spectra were simulated using TD-DFT adapted for the core hole spectra (38). All calculations were performed within the approximation of the PBE0 exchange and correlation (x-c) potential $(39,40)$, using def2-TZVP $(50)$ basis set. The TD-DFT equations were solved for 
20 states, within the Tamm Damcoff approximation and the interaction with the X-ray field was described using the electric quadrupole approximation. The transient spectrum was generated by subtracting the spectrum of the electronic ground state from a spectrum calculated using the lowest triplet state reference and shifting the energetic axis by $69.73 \mathrm{eV}$ to match the experimental trace. The lowest triplet state is used to approximate the singlet exciton in P3HT, which has the same electronic structure. This represents a good approximation due to the lack of spin sensitivity of XAS spectroscopy as shown in ref 40 . The P3HT model consisted of 6 repeat units, i.e. a P3HT hexamer. The dimer was modelled by duplicating the initial structure and displacing it $0.9 \mathrm{~nm}$ along the $z$-axis, corresponding to the $\pi$ stacking direction. All calculated spectra were broadened using a Gaussian with a fullwidth half maximum of $1.0 \mathrm{eV}$. 